\title{
Interactive comment on "Net ecosystem carbon exchange of a dry temperate eucalypt forest" by Nina Hinko-Najera et al.
}

\section{Anonymous Referee \#2}

\section{Received and published: 7 June 2016}

The carbon exchange of Eucalypt forests, which have the rare characteristic of being broadleaf evergreen, is still not well understood. This study presents the first three years of eddy covariance measurements of carbon exchange in a dry Eucalypt forest in Australia. The forest is found to be a very large sink of carbon at more than a $1000 \mathrm{~g}$ $\mathrm{C} \mathrm{m}-2 \mathrm{yr}-1$ due to yearlong GPP and a rather low ER. The main environmental drivers were assessed using a random forest analysis and the main drivers were found to be solar radiation and air temperature.

The paper brings forward some very important numbers in terms of carbon budgets for this dry temperate eucalypt forest that were missing from global flux measurements, and reveals among the largest $\mathrm{CO} 2$ sequestration for any ecosystem studied thus far. Such numbers, as well as how the fluxes vary over time (diurnal, seasonal, interannual), are highly needed to better understand how different ecosystems vary in their

Printer-friendly version

Discussion paper 
growth dynamics and ecophysiology. However, I have a series of serious concerns which I suggest need to be addressed for the paper to be considered for publication.

Main concerns:

1) On line 105, the forest height is said to be $21-27 \mathrm{~m}$, while in Table 1 it is $25-27$ $\mathrm{m}$. Which one is it? How uniform is the distribution of tree heights in the forest? The reason why this matters is that the eddy covariance (EC) system is at a height of 30 $\mathrm{m}$, thus only $3 \mathrm{~m}$ above the tallest trees. How turbulent are the winds at $30 \mathrm{~m}$ ? Did you assess whether the EC system is high enough to provide reliable fluxes or if it is located too close to the sources/sinks of fluxes and thus not well-mixed? For e.g., the wet eucalypt forest in the paper from van Gorsel et al. 2013 has a tree height of $40 \mathrm{~m}$ and the EC system is at a height of $70 \mathrm{~m}$. Why are the measurements made so close to the canopy at this site? Please show that the EC measurements are reliable at this site.

2) The flux tower is located on a ridge surrounded by some gently sloping gullies. Does the ridge affect the wind directions? Did you investigate if it leads to some advection or decoupling within the forest canopy? This is especially important for your dry eucalypt forest, as the numbers you report are extremely large for NEE while ER is low. This suggests a potential for advection within the canopy, which needs to be addressed for these numbers to be considered reliable. It is puzzling that a forest with such a low LAI sequesters so much carbon. Please consider the possible influence of advection on your fluxes. Also, please put more emphasis on the reasons why ER is so low because for the reader at the moment this is not well explained and raise skepticism on advection. How much organic matter/litter is on the soil surface? Do the eucalypt leaves ever fall? Do the trees themselves respire less than other type of trees?

3) The $u^{*}$ threshold is unusually high at 0.56 to $0.69 \mathrm{~m} \mathrm{~s}-1$. This is above most $u^{*}$ thresholds reported in the literature (see for e.g. Papale et al. 2006 Biogeoscience Fig.1) Why is it so high? Greater details need to be given on how this threshold was

Printer-friendly version

Discussion paper 
determined. Is there really an inflection point around those values? In my experience, when the common methods determine such a high threshold, it is when they do not apply i.e. there is no inflection point because it is a site with advection or with some other abnormality making the $\mathrm{u}^{*}$-threshold determination not applicable.

4) As a result of the very high $u^{*}$-threshold, only a low number of actually measured data is kept for the three years $(37 \%, 47 \%, 47 \%)$. This means that the outputted annual budgets depend mostly on the gap filling and thus, represent more modelled numbers rather than measurements. Sometimes, depending on the data quality, it can be better to use less ideal, measured data than modelled data. How different would the cumulative numbers be if for e.g. $60-70 \%$ of the measured data is kept?

5) On lines 123-125, the writing suggests that only the 30 min fluxes were stored, is that correct? If not please add more details. If it is correct, this seems like it would bias your fluxes because you cannot quality filter the raw data before flux calculations so any spikes in the raw fluxes is reflected in the 30 min fluxes. Although this cannot be changed now, it would be needed in the future to store the raw fluxes as well. In addition, did you estimate the $\mathrm{CO} 2$ storage in the canopy?

6) The authors use the random forest algorithm to determine the environmental drivers for NEE, GPP and ER. This analysis shows some serious circular argumentation. GPP is partitioned using solar radiation, thus it is beyond expected that there will be a strong relationship there. Same for ER and air temperature. Instead, I think what would help is to look at functional relationships between GPP and solar radiation, and between ER and air/soil temperature. This would provide the reader with a better understanding of how the dry eucalypt forest behaves compare to other ecosystems, as the numbers from photosynthetic capacities and such could be directly compared to those of other ecosystems. This would also add to the discussion when compared with other ecosystems to help us understand what is so different about this forest so that it sequesters so much $\mathrm{CO} 2$... At the moment the paper does not deliver clearly what makes this ecosystem so different. Improving this analysis as well as providing functional relation-

Printer-friendly version

Discussion paper 
ships figures would help to address objective 2 .

Minor comments:

Lines 51 to 57: there are four "while" in those 7 lines, including two in one sentence. Please reduce the number of "while".

Line 66: comma wrongly placed

Line 77: comma missing between abundant and there

Line 105: describe tree height better (how uniform and such) and add LAI in text.

Line 114: Is it really a mean of 114 years??

Lines 130-131: Having one soil temperature, one soil moisture and one soil heat flux plate measurement is not sufficient to characterise soil due to its spatial variability. You need replicates horizontally and vertical profiles within the soil. On line 131, SWC is at $5 \mathrm{~cm}$ depth then on line 149 you talk about $10 \mathrm{~cm}$ depth. Please clarify the soil measurement depths.

Line 144: There is an additional space before Contiuum.

Lines 145-146: Greater details need to be given on the quality filtering. This is not clear. What range checks? What thresholds for spikes? What thresholds for outliers? etc. .

Line 169: Is that how LAI was derived? Are there any ground measurements of LAI to validate the MODIS data?

Section 2.3.3: it is confusing which method you used in the end for partitioning. Please give the equations used for partitioning and show a figure comparing the different approaches if you mention you compared different methods in text. Also, show a comparison with the soil respiration measurements that you mention on line 185.

Printer-friendly version

Line 301: temperate evergreen coniferous forests (add evergreen). It is not because

Discussion paper 
they are coniferous but rather because they are evergreen.

Figure 2(a) is it NEE or -NEE (why is there a minus in the caption?) Same for Figure 3 and 4. Also, please describe what you display better, for e.g. in figure 3, what are the shaded lines at the back, the actual daily totals? Typically NEE is what is measured by $\mathrm{EC}$, and sometimes people convert it to $\mathrm{NEP}=-\mathrm{NEE}$. In your case, you do not need the minus there because you display NEE.

Interactive comment on Biogeosciences Discuss., doi:10.5194/bg-2016-192, 2016. 